\title{
Commentary
}

See article on page 845

\section{Alcoholic liver disease: dose and threshold - new thoughts on an old topic}

There is no doubt that excessive alcohol consumption leads to liver disease-from simple fatty liver to cirrhosis - in certain individuals. Questions which remain unanswered concern the relation between dose and disease risk, including the existence of a "dose threshold", the influence of sex and the precise proportion of heavy drinkers who will develop significant liver disease. Most of the relevant data have, until very recently, come from retrospective studies assessing alcohol intake in hospitalised patients at the time of diagnosis. Clearly, these studies on highly selected patients are subject to many confounding influences, ${ }^{1}$ and are also unable to provide any information on the risk of liver disease in the population stratified into drinking categories. Of the two main prospective studies that have been performed, one was confined to men and showed no dose-response relation ${ }^{2}$ and the other demonstrated no threshold effect or sex difference in disease susceptibility. ${ }^{3}$ These controversial issues have now been readdressed by two recent studies that have used different but complimentary methodological approaches, one from Becker and colleagues ${ }^{4}$ and another from Bellentani and the Dionysos Study Group reported in this issue (see page 845).

Becker et al carried out a longitudinal study of the association between self-reported alcohol intake and the risk of future liver disease in a large population based prospective cohort of 13285 subjects enrolled into the Copenhagen City Heart Study. Twelve years after enrollment the incidence of liver disease was determined from death certificates and hospital discharge records. They observed a steep dose dependent increase in relative risk of alcohol induced liver disease above a "threshold" of 7-13 drinks per week in women and 14-27 drinks per week in men. Women had a significantly higher relative risk than men for any given level of intake. Importantly, of those individuals consuming more than 70 drinks per week, only $7 \%$ were cirrhotic and only $19 \%$ had any evidence of alcohol induced liver disease at all. The main strength of this prospective longitudinal study is that it has related current alcohol intake to future disease risk. Its principal flaw concerns the method of disease ascertainment which would be expected to underestimate incidence significantly as the data were confined to deaths and inpatient hospital events.

Bellentani et al have used a different approach as part of the impressive Dionysos Study, a cross-sectional study that determined the prevalence of chronic liver disease in the entire adult population of two towns in northern Italy. ${ }^{5}$ All individuals underwent full history and examination and had limited "liver" function tests performed (alanine aminotransferase, aspartate aminotransferase, $\gamma$-glutamyl transpeptidase, mean corpuscular volume, platelet count). Patients with any clinical signs of liver disease or an abnormal blood test underwent liver ultrasound, and those with two of: stigmata of chronic liver disease, a low platelet count or abnormal ultrasound, underwent liver biopsy. All subjects completed a detailed questionnaire on alcohol intake, which was administered by a medical staff member. Individuals with anti-hepatitis $\mathrm{C}$ virus or hepatitis $\mathrm{B}$ surface antigen were excluded. Bellentani et al report a risk threshold for both non-cirrhotic and cirrhotic liver disease of 21 drinks per week in men and women with a step-wise increase in risk with increasing intake. The lifetime intake threshold for disease was $100 \mathrm{~kg}$. The risk of disease was twice as high in women than men, but only in the dose range 3-8 drinks/day. Only $4 \%$ of individuals consuming more than 6 drinks daily had cirrhosis and only $10 \%$ had any evidence of liver disease at all. The main strength of this study concerns the meticulous disease ascertainment that allows an accurate estimate to be made of the proportion of patients with liver disease within defined drinking categories. Its major flaws are, firstly, in determining cumulative alcohol intake it is assumed that current daily intake approximates to lifetime daily intake rather than adopting the alternative approach of determining complete lifetime drinking histories as suggested by Skinner and Sheu, ${ }^{6}$ and, secondly, that it is cross-sectional rather than longitudinal.

Despite the problems inherent in determining past (or future) alcohol intake from single interviews, these two studies provide by far the best evidence supporting both a threshold effect and a dose-response relation between alcohol intake and the risk of liver disease. In addition, they clearly show that, in the absence of other established cofactors, such as hepatitis viruses, only a minority (around $5 \%$ ) of heavy drinkers develop cirrhosis. Presumably, Bellentani et al will also have the opportunity to shed further light on the reported synergy between alcohol and hepatitis viruses in determining the severity of liver disease. These recent observations need to be considered in light of the current hypotheses on mechanisms of alcoholic liver disease which relate largely to the effects of ethanol metabolism generating both acetaldehyde and free radicals. Both acetaldehyde and carbon centred free radicals bind covalently to proteins to form adducts capable of initiating an immune response and altering protein function. ${ }^{7}$ Oxygen derived free radicals also induce oxidative stress which can damage hepatocytes directly, by initiating peroxidation of membrane lipids, and indirectly by stimulating transcription of pro-inflammatory cytokines including tumour necrosis factor $\alpha(\mathrm{TNF}-\alpha)$ and interleukin (IL) $8 .^{8} \mathrm{TNF}-\alpha$ may also be released in response to endotoxaemia which is common in alcoholics. One might expect that all of these postulated mechanisms would increase in intensity with increasing alcohol dose. The threshold effect presumably reflects that, below a certain level of intake, the body's intrinsic defences can cope with the insult; in particular, cellular antioxidants such as glutathione and superoxide dismutase, anti-inflammatory 
cytokines such as IL-10 and other factors responsible for limiting the magnitude of the immune response. Above this threshold, in certain individuals, the balance between disease mechanisms and these defence systems favours the development of tissue damage. Possible explanations for individual susceptibility include genetic factors influencing ethanol metabolism ${ }^{9}$ or cytokine secretion, ${ }^{10}$ and/or other environmental factors including diet. The explanation for the increased female susceptibility to disease, supported by these two studies, remains unclear but may simply reflect under-reporting by women.

The remaining, and completely new observation of the Dionysos Study, is that disease risk is determined by the pattern and type of beverage consumed. Individuals consuming alcohol with and without food and those drinking more than one type of beverage had a much higher risk of disease than individuals drinking only one type of beverage with meals. The explanation may involve the effect of food on ethanol absorption leading to a slower rise and lower peak blood alcohol concentration, but may also be due to other as yet unknown confounding factors affecting disease risk and different in drinkers with particular drinking patterns. For patients and their physicians the message seems to be drink within sensible limits and stick to your favourite tipple consumed at or around mealtimes.

C P DAY

Centre for Liver Research, Floor 4, William Leech Building, Medical

School, Framlington Place, Newcastle upon Tyne NE2 4HH, UK

1 Sørensen TIA. The relationship between alcohol consumption and risk of development of cirrhosis of the liver. Alcologia 1990; 2: 121-6.

2 Sørensen TIA, Orholm M, Bensen KD, Høybye G, Eghøje K, Christoffersen P. Prospective evaluation of alcohol abuse and alcoholic liver injury in men as predictors of development of cirrhosis. Lancet 1984; ii: 241-4.

3 Klatsky AL, Armstrong MA. Alcohol, smoking, coffee and cirrhosis. Am $\mathcal{f}$ Epidemiol 1992; 136: 1248-57.

4 Becker U, Deis A, Sørensen TIA, Grønbaek M, Borch-Johnsen K, Muller $\mathrm{CF}$, et al. Prediction of risk of liver disease by alcohol intake, sex and age: a prospective population study. Hepatology 1996; 23: 1025-9.

5 Bellentani S, Tiribelli C, Saccoccio G, Sodde M, Fratti N, DE Martin C, et al. Prevalence of chronic liver disease in the general population of Northern Italy: the Dionysos Study. Hepatology 1994; 20: 1442-9.

6 Skinner HA, Sheu S-J. Reliability of alcohol use indices: the lifetime drinking history and the MAST. $\mathcal{F}$ Stud Alcohol 1982; 43: 1157-70.

7 Israel Y. Antibodies against ethanol-derived protein adducts: pathogenic implications. Gastroenterology 1997; 113: 353-4.

8 Day CP. Is necroinflammation a prerequisite for fibrogenesis? Hepatogastroenterology 1996; 43: 104-20.

9 Day CP, James OFW, Crabb DW, Li T-K, Bassendine MF. Alcohol dehydrogenase genes and susceptibility to alcoholic liver disease. Hepatology 1993; 18: 230-232.

10 Grove J, Daly AK, Bassendine MF, Day CP. Association of a tumor necrosis factor polymorphism with susceptibility to alcoholic steatohepatitis. Hepatology 1997; 26: 143-6. 